\title{
Pre-Cataclysmic System V471 Tau with Confirmed Brown Dwarf and Suspected Extrasolar Planet
}

\author{
Ladislav Hric and Emil Kundra $\dagger$ \\ Astronomical Institute, Slovak Academy of Sciences, 05960 Tatranská Lomnica, Slovakia \\ email: hric@ta3.sk
}

\begin{abstract}
We can show that analysis of the (O-C) diagram is a powerful method of detecting new bodies in binary systems. For this purpose we need very symmetric minima with precisely determined shapes. In the case of good covering by observations with high time resolution, it is possible to determine the times of such minima with sufficient accuracy. In the case of V471 Tau, the (O-C) diagram gave us residua which can be explained by the presence of a fourth body with substellar mass in the system.
\end{abstract}

Keywords. Eclipsing binaries, cataclysmic variables, V471 Tau, exoplanets

\section{Introduction and solution}

V471 Tau is a binary pre-cataclysmic system of post-common envelope stage consisting of a white dwarf and main sequence star with an orbital period of 0.521183439 days. Existing eclipses in the system are characterized by a fast drop to the minimum as well as by a fast increase in the brightness from the minimum. The change of brightness caused by the eclipse of the white dwarf takes only 55 seconds and the particular eclipse takes only 49 minutes.

Recently we analyzed the (O-C) diagram of this system and solved the long-term problem of (O-C) diagram behaviour and model of the system (Kundra \& Hric 2010, Hric, Kundra \& Dubovský 2011). We showed that this behaviour can be explained by the presence of a third body in the system. In our case, it is a brown dwarf on an eccentric orbit with a period of 33.2 years.

Moreover, we subtracted the influence of this body from the (O-C) diagram and constructed residua (bottom panel of Fig. 1). We can see that there is still some variability in these residua and after detailed periodic analysis the resulting period is 9.3 years. In our opinion, such behaviour can be explained by another body in the system. It is worth to note that there is an alternative explanation using Applegate's mechanism. If the secondary star has a strong magnetic field with a quadruple shape, the changes of the surface of the secondary component could produce variations in the (O-C) diagram.

The output from period analysis was used as an input parameter to model the fourth body using the $3 \mathrm{~T}$ code (Pribulla et al. 2005). The results are physical and orbital parameters of the fourth body in the V471 Tau system listed in Table 1. The suspected fourth body has planetary parameters and a mass in the interval from 6 to $15 \mathrm{M}_{\mathrm{Jupiter}}$, and thus we can announce the presence of a giant extrasolar planet in this triple system.

$\dagger$ This study was supported by the project of the Slovak Academy of Sciences VEGA Grant No. $2 / 0078 / 10$. 


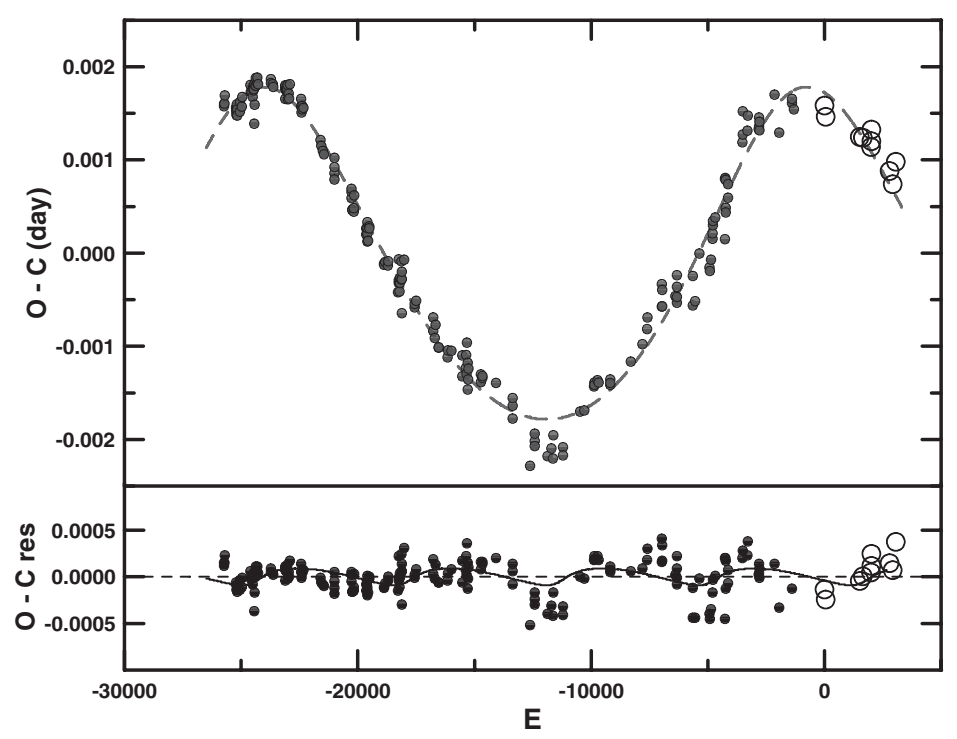

Figure 1. (O-C) diagram of V471 Tau calculated according to ephemeris of close binary. Our new observations are depicted by open circles and points represent all older minima. The bottom plot shows the residua with respect to our model of the third body with variations caused by the suspected fourth body in the system. The curve is the fit of the fourth body.

Table 1. Final physical and geometrical parameters of the fourth component of V471 Tau system. $P_{4}$ - orbital period of the fourth body, $e_{4}$ - eccentricity of the fourth component, $\omega_{4}$ argument of periastron, $T_{\text {periastron }}$ - time of periastron passage, $a_{12} \sin (i)$ - main axis orbital projection of the close binary round the center of gravity to the orbital plane; $T_{0}$ - time of minimum of the close binary selected for zero epoch, $P_{0}$ - period of close binary, $f\left(M_{4}\right)$ - mass function of the fourth component.

\begin{tabular}{ccc}
\hline Parameter & Value & Unit \\
\hline$P_{4}$ & $9.3 \pm 0.18$ & year \\
$e_{4}$ & $0.513 \pm 0.027$ & $\circ$ \\
$\omega_{4}$ & $330 \pm 10$ & $\circ$ \\
$T_{\text {periastron }}$ & $2454923 \pm 202$ & HJED \\
$a_{12} \sin (i)$ & $0.017 \pm 0.004$ & AU \\
$T_{0}$ & 2454028.452551 & HJED \\
& $\pm 4.1 \times 10^{-5}$ & \\
$P_{0}$ & 0.521183439 & day \\
& $\pm 2.01 \times 10^{-9}$ & \\
$f\left(M_{4}\right)$ & $5.99928 \times 10^{-8}$ & $\mathrm{M}_{\mathrm{Sun}}$ \\
& $\pm 1.9 \times 10^{-9}$ & \\
\hline
\end{tabular}

\section{References}

Evren, S., Ibanoglu, C., Tunca, Z., \& Tunner, O. 1985, Ap\&SSS, 120, 97

Guinan, E. F. \& Ribas, I. 2001, ApJ, 546, 43

Guinan, E. F. \& Sion, E. M. 1984, AJ, 89, 1252

Hric, L., Kundra, E., \& Dubovský, P. 2011, Contrib. Astron. Obs. Skalnaté Pleso, 41, 39

Hric, L., Petrík, K., Niarchos, P., \& Gális, R. 2003 in Stellar Astrophysics - a tribute to Helmut A. Abt, ed.: K.S. Cheng, K.C. Leung, \& T.P. Li (Dordrecht: Kluwer Cademic Publishers), Astrophysics and Space Science Library, 298, 207

Ibanoglu, C., Evren, S., Tas, G., \& Cakirlh, O. 2005 MNRAS, 360, 1077

Kamiński, K.Z., Ruciński, S.M., Matthews, J.M., Kuschnig, R., Rowe, J.F., Guenther, D.B., Mofat, A.F.J., Sasselov, D., Walker, G.A.H., \& Weiss, W.W. 2007 AJ, 134, 1206

Kundra, E. \& Hric, L. 2010, Ap\&SS, 331, 121

Pribulla, T., Chochol, D., Tremko, J., \& Kreiner, J.M. 2005 in The Light-Time Effect in Astrophysics, ed.: C. Sterken, PASP, 103 